\title{
Star Formation History of Dwarf Galaxies in Cosmological Hydrodynamic Simulations
}

\author{
Kentaro Nagamine \\ Department of Physics and Astronomy, University of Nevada, Las Vegas 4505 S. Maryland Pkwy, \\ Box 454002, Las Vegas, NV 89154-4002, USA \\ Correspondence should be addressed to Kentaro Nagamine, kn@physics.unlv.edu
}

Received 8 May 2009; Revised 31 August 2009; Accepted 30 September 2009

Academic Editor: Andrey V. Kravtsov

Copyright (๑) 2010 Kentaro Nagamine. This is an open access article distributed under the Creative Commons Attribution License, which permits unrestricted use, distribution, and reproduction in any medium, provided the original work is properly cited.

We examine the past and current work on the star formation (SF) histories of dwarf galaxies in cosmological hydrodynamic simulations. The results obtained from different numerical methods are still somewhat mixed, but the differences are understandable if we consider the numerical and resolution effects. It remains a challenge to simulate the episodic nature of SF history in dwarf galaxies at late times within the cosmological context of a cold dark matter model. More work is needed to solve the mysteries of SF history of dwarf galaxies employing large-scale hydrodynamic simulations on the next generation of supercomputers.

\section{Introduction}

Dwarf galaxies play unique roles in the cosmological studies of galaxy formation. Low-mass galaxies contribute the most to the cosmic star formation rate (SFR) density at low redshift, and in contrast the star formation in high-mass galaxies seems to cease at $z \gtrsim 1[1,2]$ : the so-called "downsizing" effect in galaxy formation. This global trend of star formation is presumably driven by the feedback effects by supernovae $(\mathrm{SNe})$ and supermassive black holes as well as the cosmological effects such as the expansion and the reionization of the Universe. All of these effects can suppress the star formation, eventually giving rise to the characteristic shape of galaxy luminosity function. The question that is not fully answered yet is "which physical processes have the strongest impact, and which galaxies are affected the most?" Studying the star formation in dwarf galaxies gives us useful clues not only on the physics of star formation but also on the feedback processes that cause the downsizing in galaxy formation and how the galaxy luminosity function has been shaped over time.

Observations of stellar populations suggest that the star formation in dwarf galaxies [3-7] is sporadic, separated by millions to billions of years, even in isolated systems. What causes the episodic SF history? Past interaction with other galaxies or merger events are obvious possibilities to explain the SF activity in these systems, but the lack of tidal debris in the outer regions of dwarf galaxies argues against such scenarios [8]. Then the remaining likely possibility is the instability in the local interstellar medium (ISM); however, many interesting questions remain. For example, why is it now that they are undergoing active star formation? What determines the epoch and the duration of star formation? Is it the local physics or the cosmological processes that are more important in determining the downsizing effect?

While the recent episodic SF activity is observed in dwarf galaxies, most dwarf galaxies in the Local Group are also dominated by the old stellar populations (ages of $\gtrsim 10 \mathrm{Gyr}$ ) with an occasional mixture of younger stars [9-12]. This suggests that the main formation epoch of those dwarf galaxies was before $z \sim 6$.

It is important to address the above questions using the ab initio cosmological hydrodynamic simulations, in which the gas dynamics is simulated self-consistently from early universe to the present time. Although there has been much numerical work on the formation of dwarf galaxies [13-20], usually the work is presented in the context of reionization or the missing satellite problem, and most publications do not present the SF histories of dwarf galaxies in their simulations. 
As we describe in the next section, explaining the episodic nature of SF history in low-mass systems remains a challenge for many reasons in the framework of cold dark matter (CDM) model. The purpose of this short article is to record what we know on the SF history of dwarf galaxies in the past and current cosmological hydrodynamic simulations.

\section{Cosmological Hydrodynamic Simulations}

2.1. Star Formation Model. In order to simulate the formation and evolution of dwarf galaxies, one has to model the collapse of gas clouds and subsequent star formation. In most cosmological simulations, the star formation is modeled by creating a star particle from a parcel of gas (either the gas in an Eulerian cell or a gas particle in the case of smoothed particle hydrodynamics $[\mathrm{SPH}]$ ) when the following criteria are satisfied [21, 22]: (i) the region is overdense $\left(\delta>\delta_{c}\right)$, (ii) converging gas flow $(\nabla \cdot \mathbf{v}<0)$, (iii) cooling rapidly $\left(t_{\text {cool }}<t_{\text {dyn }}\right)$, and (iv) Jeans unstable $\left(m_{\text {gas }}>m_{\text {Jeans }}\right)$. One would expect that if the condition (iii) or (iv) is satisfied, the other conditions are also likely to be met, but more rigorous analyses would be required to assess which criterion is the most important one. There are other variants of SF models, but the discussion will be deferred to another place [23].

Simulations of galaxy formation require vast dynamic ranges in both mass and space: from molecular clouds ( $\sim$ parsecs $)$ to groups/clusters of galaxies ( $\sim$ mega-parsecs). With the currently available computational resources, it is still difficult to resolve the details of molecular clouds while simultaneously simulating the formation of thousands of galaxies on the scales of $\gtrsim 10 \mathrm{Mpc}$. In other words, we can reliably identify the sites of galaxy formation on large scales and simulate the average SFR of each galaxy, but the smallscale instabilities of multiphase ISM on subparsec scales caused by, for example, turbulence, cannot be followed in detail in cosmological simulations. As we will describe in the next section in more detail, the earlier Eulerian cosmological simulation [24] was not able to reproduce the episodic nature of SF history, even though it properly simulated the gas dynamics on large scales ( $20 \mathrm{kpc}$ ). This suggests that the sporadic star formation in dwarf galaxies is driven by the instabilities on small scales, which is difficult to simulate properly with the current resolution limits.

The above limitation is a typical criticism directed towards cosmological simulations; however, the cosmological simulations can calculate the amount of gas that fall into the region correctly. Combined with a star formation law, we can calculate the overall SFR of the system, which is valid within the limitations of the model and still is relevant to the subject of this article. We simulate the gas dynamics at intermediate scales $(\sim \mathrm{kpc})$ as accurately as possible and treat the small-scale physics with a subgrid/particle model for star formation and SN feedback based on our astrophysical knowledge. In this approach, the SFR is calculated as $\dot{\rho}_{\star}=c_{\star}\left(\rho_{\text {gas }} / t_{\star}\right)$, where $c_{\star}$ is the SF efficiency and $t_{\star}$ is the SF time-scale. Both of these parameters are usually adjusted so that the simulation result matches the observed Kennicutt-Schmidt law $[25,26]$. One can further adopt a subgrid/particle model $[27,28]$ to calculate the cold gas density $\rho_{\text {cold }}$ and use this in place of $\rho_{\text {gas }}$ in the above SF law. The projected SFR generally follows the Kennicutt law well $[27,29-31]$, but the consequences of the adopted SF model (e.g., the SF threshold density or the equation of state) must be studied further [23].

2.2. Past and Current Work. The work in [24] showed that, using an Eulerian cosmological hydrodynamic simulation, the star formation ceased at high redshift in the dwarf galaxies that survived to $z=0$. In their simulation, only $7 \%$ of stars formed below $z=1$ in galaxies with stellar masses $2 \times 10^{8}<M_{\star}(z=0)<2 \times 10^{9} h^{-1} M_{\odot}$, and no stars formed at $z<1$ in galaxies with $M_{\star}(z=0)<2 \times 10^{8} h^{-1} M_{\odot}$. This result is consistent with the general expectation in the CDM model, in which the small systems form early on and the accretion of material stops thereafter, as the residual gas is easily swept out by the SN feedback or evaporated by photoionization $[13,32-36]$. The conclusion of [24] was that, in a CDM universe, dwarf galaxies found today are dominated by old stars; they consist predominantly of stars 10 Gyrs old and do not show recent SF activity.

However, as we outlined in Section 1, the observed SF histories of dwarf galaxies exhibit sporadic SF activity at late times, and we wonder whether the result of [24] was affected by the resolution limitation. Eulerian mesh simulations lose the physical spatial resolution at late times owing to the cosmic expansion. On the other hand, they generally have a larger mesh number compared to the number of particles employed in cosmological SPH simulations; hence they have higher baryonic mass resolution at early times than $\mathrm{SPH}$ simulations. (If the number of dark matter particles and the box sizes are comparable, then the resolution in the initial gravitational field would be similar in the two methods.) This numerical trend could explain the efficient conversion of gas into stars at early times and the lack of SF activity at late times in the Eulerian mesh simulation of [24], because the simulation may underestimate the cooling rate of gas when it cannot resolve the density fluctuation of gas on scales below the physical mesh size, as they approach to the present time. A related notable advantage of the Eulerian mesh simulation over the SPH method is that it is better at modeling the gas in low-density regions; therefore it is more suitable for the study of Ly- $\alpha$ forest [37].

In contrast to the Eulerian mesh simulation, the SPH simulation is more suitable to simulate the late-time evolution of high-density regions owing to its Lagrangian nature. Therefore one might expect that the SPH simulations perform better in modeling the sporadic SF history at late times in dwarf galaxies. In the left panel of Figure 1, we compare some examples of SF history in a cosmological SPH simulation of a comoving box size $L_{\text {box }}=100 \mathrm{~h}^{-1} \mathrm{Mpc}$ and $2 \times 400^{3}$ (gas and dark matter) particles. The adopted cosmological parameters are consistent with the latest WMAP5 results [38]: $\left(\Omega_{\mathrm{m}}, \Omega_{\Lambda}, \Omega_{\mathrm{b}}, \sigma_{8}, h\right)=(0.26,0.74,0.044,0.80,0.72)$, where $h=H_{0} /\left(100 \mathrm{~km} \mathrm{~s}^{-1} \mathrm{Mpc}^{-1}\right)$. The masses of dark matter, gas, and star particles are $\left(m_{\mathrm{dm}}, m_{\mathrm{gas}}, m_{\mathrm{star}}\right)=(9.4 \times$ $\left.10^{8}, 1.9 \times 10^{8}, 8.5 \times 10^{7}\right) h^{-1} M_{\odot}$. In each panel, we indicate the 


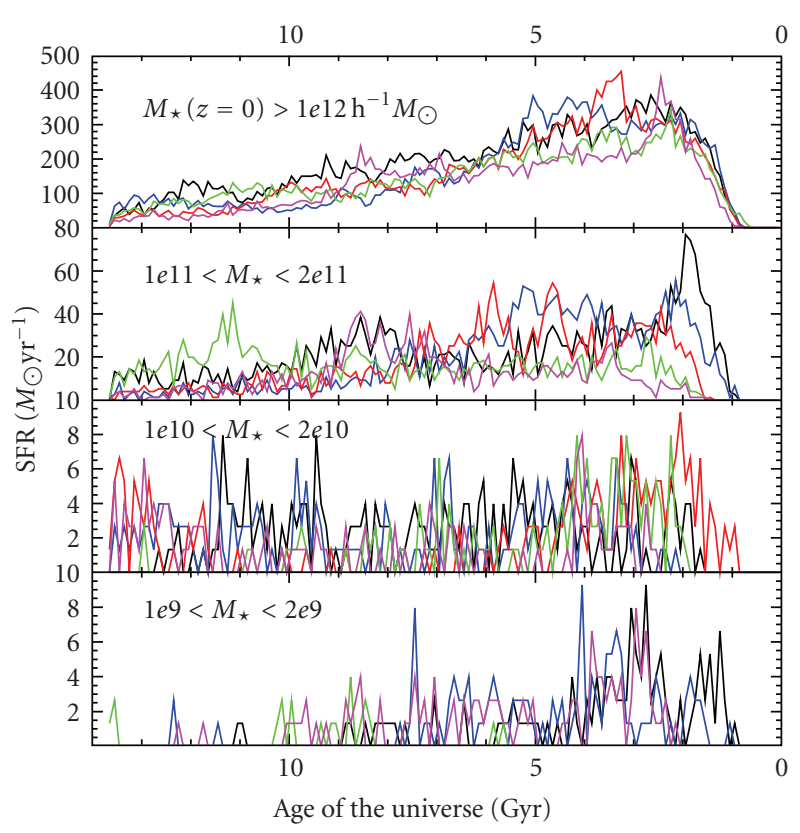

(a)

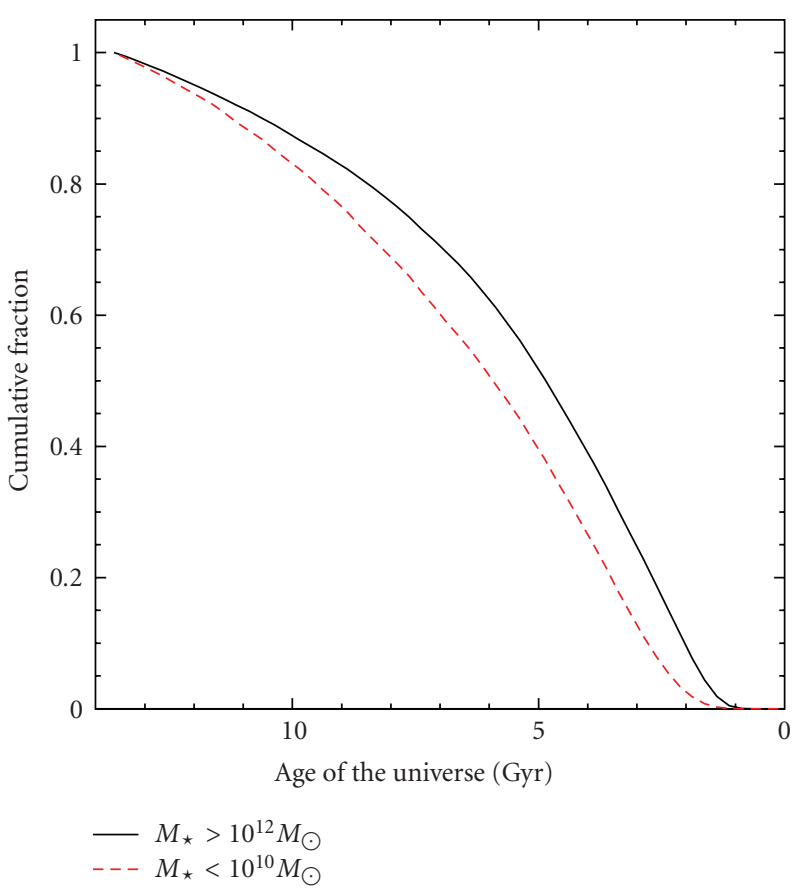

(b)

FIGURE 1: (a) We compare some examples of star formation histories with $10^{8} \mathrm{yr}$ bins in a cosmological SPH simulation of a comoving box size $L_{\text {box }}=100 h^{-1} \mathrm{Mpc}$ and $2 \times 400^{3}$ (gas and dark matter) particles. In each panel, five different galaxies in the indicated stellar mass ranges (in units of $h^{-1} M_{\odot}$ ) at $z=0$ are shown with different colors. Stars formed in all the progenitors are coadded for each galaxy. (b) Cumulative stellar mass fraction as a function of cosmic time for all the galaxies with $M_{\star}(z=0)>10^{12} h^{-1} M_{\odot}$ and $M_{\star}(z=0)<10^{10} h^{-1} M_{\odot}$.

range of stellar masses of the selected galaxies at $z=0$. Each SF history includes all the stars formed in the progenitors of the current galaxy. The SF histories of the most massive galaxies with $M_{\star}>10^{12} h^{-1} M_{\odot}$ peak at $t=2-3 \mathrm{Gyr}$ and gradually decline thereafter in a roughly exponential manner, consistently with the results of [24]. In the bottom panel, the SF histories of dwarf galaxies with $1 \times 10^{9}<$ $M_{\star}<2 \times 10^{9} h^{-1} M_{\odot}$ are shown. In this simulation, the dwarf galaxies continue to form stars sporadically at late times, even at $t>10$ Gyrs.

In the right panel of Figure 1, we compare the cumulative stellar mass fraction that formed in galaxies with $M_{\star}(z=$ $0)>10^{12} h^{-1} M_{\odot}$ and $M_{\star}(z=0)<10^{10} h^{-1} M_{\odot}$ as a function of the age of the universe. This figure shows that, in a relative sense, the more massive galaxies form their stars earlier than the lower mass galaxies, in qualitative agreement with the observational trend (e.g., [39]) of downsizing.

Even though our new SPH simulations [23] seem to be more successful in modeling the qualitative trend of star formation in different galaxies, we may still have difficulties in reproducing the correct number density of "red and dead" massive galaxies at $z=1-2$ or the ultraluminous infrared galaxies (ULIRGs), as examined in [40, 41]. Interestingly, their work showed that the Eulerian simulation exhibited more sporadic SF history for the massive galaxies than the SPH simulation at intermediate redshifts of $z=1-3$. The difference in the nature of SF history was perhaps due to a combination of differences in the SF models and the effectiveness of feedback as well as the numerical resolution reached in the different simulations.

\section{Discussion and Conclusion}

As described in the previous section, the results from different numerical methods are still somewhat mixed, but we understand their qualitative differences when we consider the resolution and numerical effects. The main factor seems to be the numerical resolution, and one way to get around this problem might be to utilize the adaptive mesh refinement (AMR) technique (e.g., [20]). However, as we pointed out in [42], current AMR codes require substantially larger computational resources to obtain an equivalent result on the dark matter halo mass function as the SPH codes do at early times. Therefore it is difficult to simulate a large sample of dwarf galaxies in a large cosmological volume, and the simulation is usually not run down to $z=0$ $[20,43]$.

Perhaps the best way to view the current situation is that each code captures the essence of dwarf galaxy formation at different epochs. The Eulerian mesh codes can capture the very early starbursts in low-mass halos better than the SPH codes, whereas the SPH codes can capture the gas infall and star formation in dwarf galaxies at late times owing to their higher spatial resolution in high-density regions. Both of these processes (i.e., early and late star formation) probably 
took place in the real Universe as [18] proposed: some dwarf galaxies being the true fossils of the pre-reionization era, some being dominated by the late star formation at low redshift, and the rest being the mixture of the two.

Our hope is that, within the next decade, the results from different numerical techniques (Eulerian mesh, SPH, and AMR) will converge and provide a consistent picture of dwarf galaxy formation. Then we will have a much better idea on the physical processes that shaped the galaxy luminosity function and how the downsizing effect of galaxy formation is caused. To achieve this goal, we still have to overcome a huge dynamic range from subparsec to Mpc scales, and it will require a petascale supercomputer of next generation, such as Blue Waters [44].

\section{Acknowledgments}

The author is grateful to Jun-Hwan Choi who kindly allowed him to present the results of his cosmological SPH simulations that he is collaborating on. The full results of our work will be presented elsewhere [23]. This work is supported in part by the National Science Foundation Grant AST0807491, the National Aeronautics and Space Administration under Grant/Cooperative Agreement no. NNX08AE57A issued by the Nevada NASA EPSCoR program, the National Science Foundation through TeraGrid resources provided by the Texas Advanced Computing Center and the San Diego Supercomputer Center, and the President's Infrastructure Award at UNLV.

\section{References}

[1] S. Juneau, K. Glazebrook, D. Champton, et al., "Cosmic star formation history and its dependence on galaxy stellar mass," The Astrophysical Journal, vol. 619, no. 2, pp. L135-L138, 2005.

[2] E. Le Floc'h, C. Papovich, H. Dole, et al., "Infrared luminosity functions from the chandra deep field-south: the Spitzer view on the history of dusty star formation at $0<z<1$," Astrophysical Journal, vol. 632, no. 1, pp. 169-190, 2005.

[3] A. E. Dolphin, A. Saha, E. D. Skillman, et al., "Deep hubble space telescope imaging of sextans A. III. The star formation history," Astronomical Journal, vol. 126, pp. 187-196, 2003.

[4] A. E. Dolphin, D. R. Weisz, E. Skillman, et al., "Star formation histories in local group dwarf galaxies," New Astronomy Reviews, vol. 49, no. 7-9, pp. 453-460, 2005.

[5] K. B. W. McQuinn, E. D. Skillman, J. M. Cannon, et al., "The true durations of starbursts: hubble space telescope observations of three nearby dwarf starburst galaxies," The Astrophysical Journal, vol. 695, pp. 561-573, 2009.

[6] L. Searle, W. L. W. Sargent, and W. G. Bagnuolo, "The history of star formation and the colors of late-type galaxies," The Astrophysical Journal, vol. 179, pp. 427-438, 1973.

[7] D. R. Weisz, E. D. Skillman, J. M. Cannon, et al., "The recent star formation histories of M81 group dwarf irregular galaxies," The Astrophysical Journal, vol. 689, no. 1, pp. 160183,2008

[8] K. E. Dellenbusch, J. S. Gallagher III, P. M. Knezek, and A. G. Noble, "Deep optical imaging of starbursting "transition" dwarf galaxies," The Astronomical Journal, vol. 135, no. 1, pp. 326-332, 2008.
[9] N. Y. Gnedin, "Local group dwarf galaxies and the star formation law at high redshift," The Astrophysical Journal, vol. 535, no. 2, pp. L75-L78, 2000.

[10] E. K. Grebel, "Star formation histories of local group dwarf galaxies," Reviews in Modern Astronomy, vol. 10, pp. 29-60, 1997.

[11] M. Mateo, "Dwarf galaxies of the Local Group," Annual Review of Astronomy and Astrophysics, vol. 36, no. 1, pp. 435-506, 1998.

[12] S. van den Bergh, "The local group of galaxies," Astronomy and Astrophysics Review, vol. 9, no. 3-4, pp. 273-318, 1999.

[13] N. Y. Gnedin, "Effect of reionization on structure formation in the universe," The Astrophysical Journal, vol. 542, pp. 535-541, 2000.

[14] N. Y. Gnedin and A. V. Kravtsov, "Fossils of reionization in the local group," The Astrophysical Journal, vol. 645, no. 2, pp. 1054-1061, 2006.

[15] M. Hoeft, G. Yepes, S. Gottlöber, and V. Springel, "Dwarf galaxies in voids: suppressing star formation with photoheating," Monthly Notices of the Royal Astronomical Society, vol. 371, no. 1, pp. 401-414, 2006.

[16] A. V. Kravtsov, O. Y. Gnedin, and A. A. Klypin, "The tumultuous lives of galactic dwarfs and the missing satellites problem," The Astrophysical Journal, vol. 609, no. 2, pp. 482497, 2004.

[17] S. Mashchenko, H. M. P. Couchman, and A. Sills, "Modeling star formation in dwarf spheroidal galaxies: a case for extended dark matter halos," The Astrophysical Journal, vol. 624, no. 2, pp. 726-741, 2005.

[18] M. Ricotti and N. Y. Gnedin, "Formation histories of dwarf galaxies in the Local Group," The Astrophysical Journal, vol. 629, no. 1, pp. 259-267, 2005.

[19] K. Tassis, A. V. Kravtsov, and N. Y. Gnedin, "Scaling relations of dwarf galaxies without supernova-driven winds," The Astrophysical Journal, vol. 672, no. 2, pp. 888-903, 2008.

[20] K. Tassis, T. Abel, G. L. Bryan, and M. L. Norman, "Numerical simulations of high-redshift star formation in dwarf galaxies," The Astrophysical Journal, vol. 587, no. 1, pp. 13-24, 2003.

[21] R. Cen and J. P. Ostriker, "Galaxy formation and physical bias," The Astrophysical Journal, vol. 399, no. 2, part 2, pp. L113L116, 1992.

[22] N. Katz, "Dissipational galaxy formation. II. Effects of star formation," The Astrophysical Journal, vol. 391, no. 2, pp. 502$517,1992$.

[23] J.-H. Choi and K. Nagamine, "Effects of cosmological parameters and star formation models on the cosmic star formation history in LambdaCDM cosmological simulations," Monthly Notices of the Royal Astronomical Society, http://lanl.arxiv.org/abs/0909.5425.

[24] K. Nagamine, M. Fukugita, R. Cen, and J. P. Ostriker, "Star formation history and stellar metallicity distribution in a cold dark matter universe," The Astrophysical Journal, vol. 558, no. 2, pp. 497-504, 2001.

[25] R. C. Kennicutt Jr., "The global schmidt law in star-forming galaxies," The Astrophysical Journal, vol. 498, pp. 541-552, 1998.

[26] M. Schmidt, "The rate of star formation," The Astrophysical Journal, vol. 129, p. 243, 1959.

[27] V. Springel and L. Hernquist, "Cosmological smoothed particle hydrodynamics simulations: a hybrid multiphase model for star formation," Monthly Notices of the Royal Astronomical Society, vol. 339, no. 2, pp. 289-311, 2003.

[28] G. Yepes, R. Kates, A. Khokhlov, and A. Klypin, "Hydrodynamical simulations of galaxy formation: effects of supernova 
feedback," Monthly Notices of the Royal Astronomical Society, vol. 284, no. 1, pp. 235-236, 1997.

[29] J.-H. Choi and K. Nagamine, "Effects of metal enrichment and metal cooling in galaxy growth and cosmic star formation history," Monthly Notices of the Royal Astronomical Society, vol. 393, no. 4, pp. 1595-1607, 2009.

[30] K. Nagamine, V. Springel, and L. Hernquist, "Star formation rate and metallicity of damped Lyman $\alpha$ absorbers in cosmological smoothed particle hydrodynamics simulations," Monthly Notices of the Royal Astronomical Society, vol. 348, no. 2, pp. 435-450, 2004.

[31] J. Schaye and C. Dalla Vecchia, "On the relation between the Schmidt and Kennicutt-Schmidt star formation laws and its implications for numerical simulations," Monthly Notices of the Royal Astronomical Society, vol. 383, no. 3, pp. 1210-1222, 2008.

[32] A. Dekel and J. Silk, "The origin of dwarf galaxies, cold dark matter, and biased galaxy formation," The Astrophysical Journal, vol. 303, part1, pp. 39-55, 1986.

[33] G. Efstathiou, "Suppressing the formation of dwarf galaxies via photoionization," Monthly Notices of the Royal Astronomical Society, vol. 256, no. 2, pp. 43P-47P, 1992.

[34] J. F. Navarro and M. Steinmetz, "The effects of a photoionizing ultraviolet background on the formation of disk galaxies," The Astrophysical Journal, vol. 478, no. 1, pp. 13-28, 1997.

[35] T. Quinn, N. Katz, and G. Efstathiou, "Photoionization and the formation of dwarf galaxies," Monthly Notices of the Royal Astronomical Society, vol. 278, no. 4, pp. L49-L54, 1996.

[36] D. H. Weinberg, L. Hernquist, and N. Katz, "Photoionization, numerical resolution, and galaxy formation," The Astrophysical Journal, vol. 477, no. 1, pp. 8-20, 1997.

[37] R. Cen, J. Miralda-Escudé, J. P. Ostriker, and M. Rauch, "Gravitational collapse of small-scale structure as the origin of the Lyman-alpha forest," The Astrophysical Journal, vol. 437, no. 1, part 2, pp. L9-L12, 1994.

[38] E. Komatsu, J. Dunkley, M. R. Nolta, et al., "Five-year wilkinson microwave anisotropy probe observations: cosmological interpretation," The Astrophysical Journal, Supplement Series, vol. 180, no. 2, pp. 330-376, 2009.

[39] D. Thomas, C. Maraston, R. Bender, and C. M. de Oliveira, "The epochs of early-type galaxy formation as a function of environment," The Astrophysical Journal, vol. 621, no. 2, pp. 673-694, 2005.

[40] K. Nagamine, R. Cen, L. Hernquist, J. P. Ostriker, and V. Springel, "Massive galaxies and extremely red objects at $z=$ $1-3$ in cosmological hydrodynamic simulations: near-infrared properties," The Astrophysical Journal, vol. 627, no. 2, pp. 608620, 2005.

[41] K. Nagamine, R. Cen, L. Hernquist, J. P. Ostriker, and V. Springel, "Massive galaxies in cosmological simulations: ultraviolet-selected sample at redshift $z=2$," The Astrophysical Journal, vol. 618, pp. 23-37, 2005.

[42] B. W. O'Shea, K. Nagamine, V. Springel, L. Hernquist, and M. L. Norman, "Comparing AMR and SPH cosmological simulations. I. Dark matter and adiabatic simulations," The Astrophysical Journal, Supplement Series, vol. 160, no. 1, pp. 127, 2005.

[43] N. Y. Gnedin, K. Tassis, and A. V. Kravtsov, "Modeling molecular hydrogen and star formation in cosmological simulations," The Astrophysical Journal, vol. 697, no. 1, pp. 5567, 2009.

[44] http://www.ncsa.illinois.edu/BlueWaters/. 

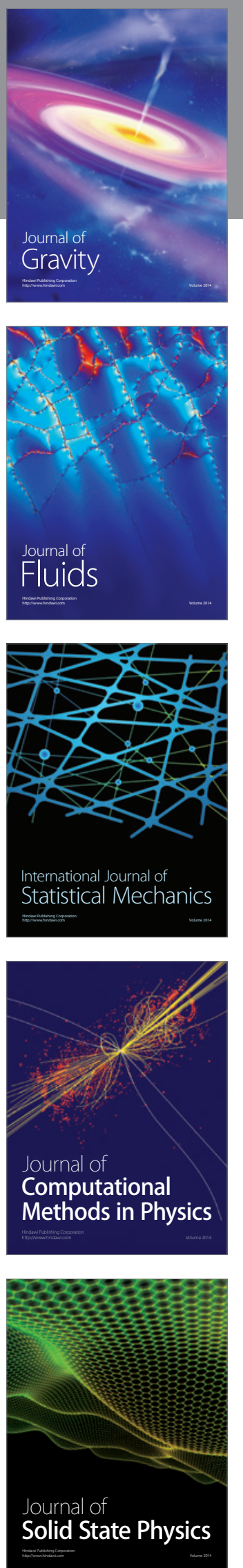

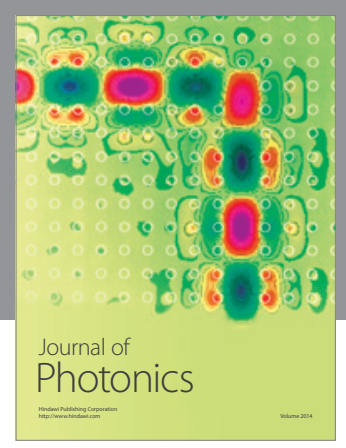

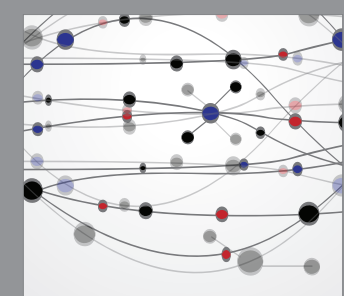

The Scientific World Journal
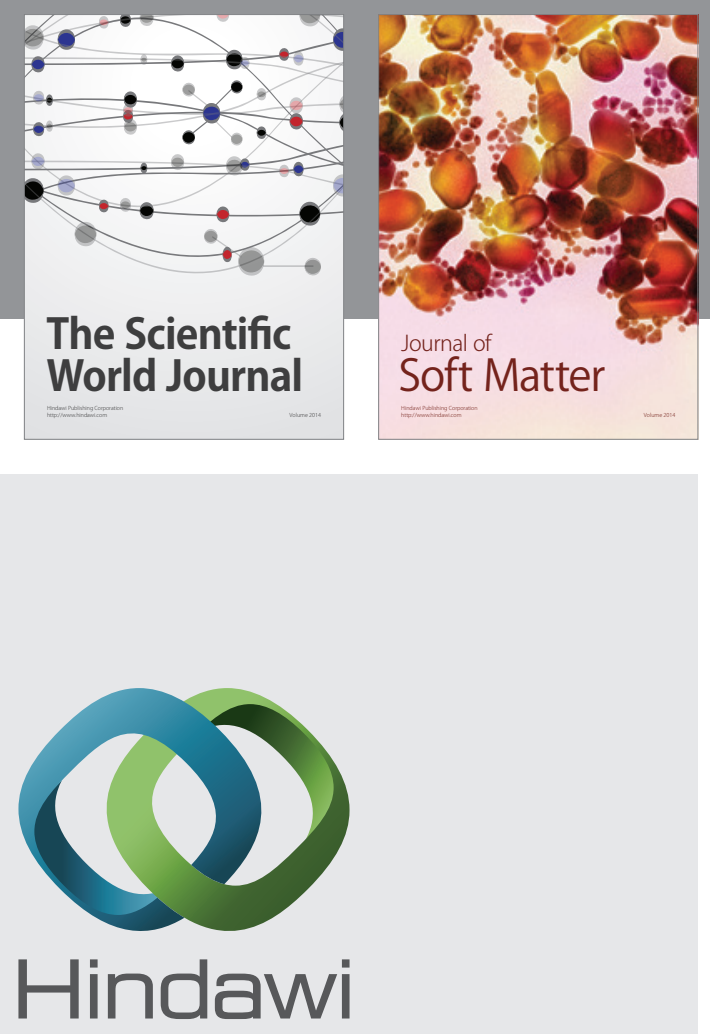

Submit your manuscripts at

http://www.hindawi.com
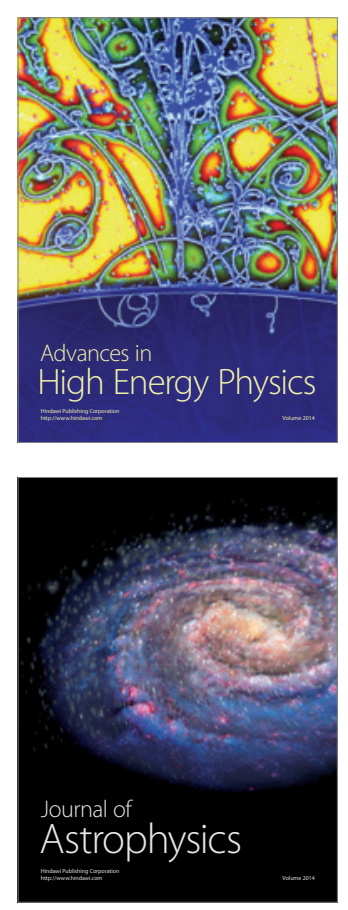
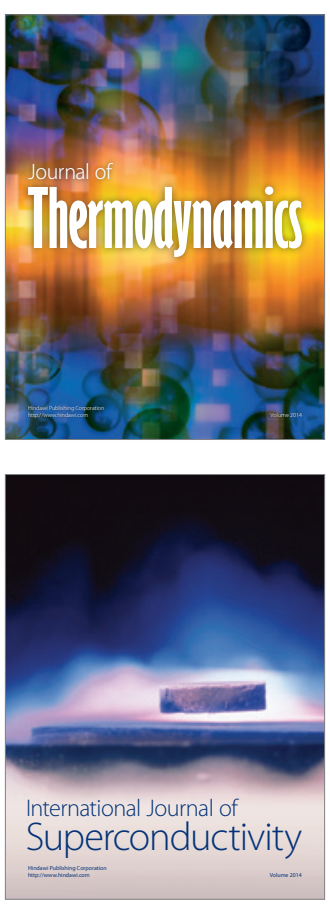
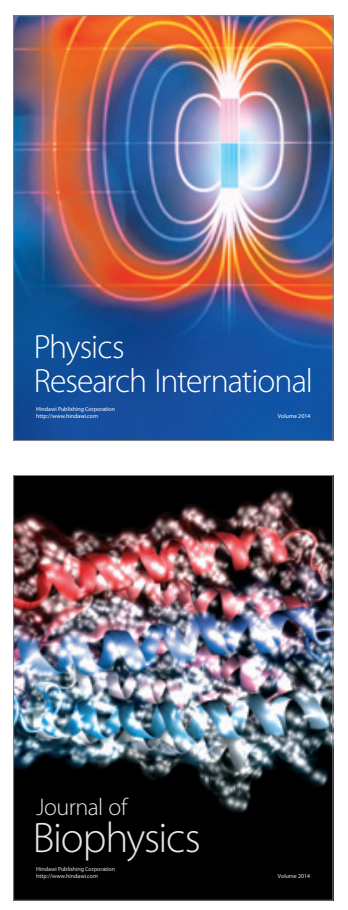
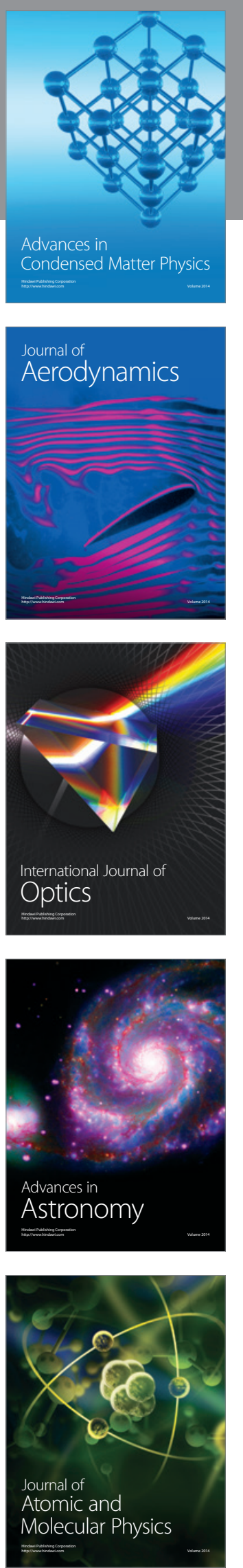